\title{
What does a mouse tell us about neuregulin 1-cannabis interactions?
}

\author{
Tim Karl ${ }^{1,2,3 *}$ and Jonathon C. Arnold ${ }^{2,4,5}$ \\ ${ }^{1}$ Neuroscience Research Australia, Randwick, NSW, Australia \\ 2 Schizophrenia Research Institute, Darlinghurst, NSW, Australia \\ ${ }^{3}$ School of Medical Sciences, University of New South Wales, NSW, Australia \\ ${ }^{4}$ Department of Pharmacology, Bosch Institute, University of Sydney, NSW, Australia \\ ${ }^{5}$ Brain and Mind Research Institute, Camperdown, NSW, Australia
}

\section{Edited by:}

Chao Deng, University of

Wollongong, Australia

Reviewed by:

Satpal Singh, State University of

New York at Buffalo, USA

Corette Wierenga, Utrecht

University, Netherlands

\section{*Correspondence:}

Tim Karl, Neuroscience Research

Australia, Barker St., Randwick,

NSW 2031, Australia.

e-mail: t.kar@@eura.edu.au
The link between cannabis and psychosis has been debated although there is substantial epidemiological evidence showing that cannabis increases the risk of psychosis. It has been hypothesized that schizophrenia patients carrying particular risk genes might be more sensitive to the psychosis-inducing effects of cannabis than other patients and healthy test subjects. Here we review the effects of cannabinoids on a mutant mouse model for the schizophrenia candidate gene neuregulin 1 (Nrg1). The studies suggest a complex interaction between cannabis and Nrg1: the neuro-behavioral effects of cannabinoids were different in Nrg1 mutant and control mice and depended on exposure time, sex, and age of test animals. This research provides the first evidence of complex cannabis-Nrg1 interactions suggesting $\mathrm{Nrg} 1$ as a prime target for future clinical investigations. Furthermore, it highlights that animal model research can broaden our understanding of the complex multi-factorial etiology of schizophrenia. Finally, the findings are important to preventive psychiatry: if the genes that confer genetic vulnerability to cannabis-induced psychosis were identified patients at-high risk could be forewarned of the potential dangers of cannabis abuse.

Keywords: schizophrenia, cannabis, neuregulin 1, gene-environment interactions, mouse model
The two-hit hypothesis of schizophrenia states that a combination of genetic and environmental risk factors will cause the development of schizophrenia (Bayer et al., 1999; Rapoport et al., 2005; Caspi and Moffitt, 2006). Scores of genetic risk factors have been suggested for schizophrenia (Allen et al., 2008) and mouse mutants have been developed for most of those candidates (Desbonnet et al., 2009). Neuregulin 1 (NRG1) is one of the more promising schizophrenia candidate genes as associations with schizophrenia have been found in several studies (Stefansson et al., 2002; Tosato et al., 2005; Munafo et al., 2006). However, similar to many other schizophrenia susceptibility genes, recent genome wide associations studies suggest that it is more important to consider an interplay of different genetic and environmental risk factors for schizophrenia to understand the etiology of the disorder (Sanders et al., 2008). Thus, many environmental factors have been considered as risk factors for schizophrenia (Van Os et al., 2010) and cannabis use has been the focus of an ever larger growing list of studies. Cannabis appears to be a component/cumulative cause for schizophrenia and increases the overall risk of developing the disorder by 2 -fold (Henquet et al., 2005). Importantly, an increasing number of researchers believe that this risk might be elevated for cannabis users with a genetic vulnerability to schizophrenia (Caspi and Moffitt, 2006). Indeed, a functional polymorphism in the gene for catechol-O-methyl transferase $(C O M T)$ was implicated in conferring vulnerability to cannabis-induced psychosis (Caspi et al., 2005; Henquet et al., 2006). Subsequently, a genetic mouse model for COMT was treated with the main psychoactive component of cannabis ( $\Delta^{9}$-tetrahydrocannabinol: THC) during adolescence and exhibited a greater behavioral sensitivity to the long-term effects of THC (O'Tuathaigh et al., 2010) and a genotype-specific response in dopaminergic and GABAergic pathways as well as in the protein expression of cannabinoid 1 receptors (CB1) (Behan et al., 2012). Based on NRG1's established role in schizophrenia and the availability of validated mouse mutants for Nrg1 (Duffy et al., 2008), we investigated over the last decade if $\mathrm{Nrg1}$ represents a second candidate for gene-cannabis interactions in schizophrenia. The mini review will outline how our mouse research has been instrumental in discovering $\mathrm{Nrgl}$-cannabis interactions relevant to schizophrenia and in deciphering potential mechanisms. Our studies not only considered THC but also the cannabinoid cannabidiol (CBD), which is devoid of psychoactive properties and has been reported to block or reverse effects of THC and have antipsychotic properties (Arnold et al., 2012).

The protein Nrg1 influences key neurodevelopmental processes such as myelination, synaptogenesis, neuronal migration, and is involved in the expression and activation of N-methyl-Daspartic acid (NMDA) receptors (Harrison and Law, 2006; Mei and Xiong, 2008). Importantly, a number of genetic mouse models have been developed for the different isoforms of Nrg1 (Duffy et al., 2008; Mei and Xiong, 2008; Karl et al., 2011). Among those, the heterozygous transmembrane domain Nrg1 mutant mouse (Nrg1 HET) has shown compelling face, construct, and partial predictive validity for schizophrenia research (Stefansson 
et al., 2002; Karl et al., 2007, 2011; Van Den Buuse et al., 2009; Duffy et al., 2010; Chesworth et al., 2012a,b). Thus, our team has utilized this model to determine the nature of Nrg1-cannabis interactions in great detail (for review see Arnold et al., 2012). The clinical relevance of this research has recently been highlighted by a genome-wide linkage and single nucleotide polymorphism association analysis, which discovered NRG1 as a major candidate for the development of cannabis dependence in African Americans (Han et al., 2012). The findings of our earlier mouse model research will be outlined in the following.

In an initial study we exposed Nrg1 HET mice to acute doses of THC before testing them in an array of schizophreniarelevant behavioral paradigms (Powell and Miyakawa, 2006). Nrg1 mutant mice exhibited an increased sensitivity to the locomotor-suppressant and anxiogenic effects of THC compared to wild type-like littermates (WT). Surprisingly, the mutants also showed improved sensorimotor gating following THC challenge as measured by prepulse inhibition of the startle response (PPI) (Boucher et al., 2007a). Increased PPI is often detected after treatment with antipsychotic drugs which normalize PPI deficits of schizophrenia patients (Geyer et al., 2001). Recent human data suggest that NRG1 may also confer increased behavioral sensitivity to THC [although NRG1 polymorphisms worsened THC-induced information processing dysfunction rather than improving it (Stadelmann et al., 2010)]. It is possible that the effects of heterozygous deletion of Nrgl in mice might be opposite to that conferred by NRG1 polymorphisms in patients. Future studies may examine whether mice overexpressing Nrg1 protein display exaggerated THC-induced PPI deficits. Followup experiments revealed that the enhanced behavioral response of Nrg1 HETs to acute THC was sex-specific as female mutants showed no enhanced susceptibility to acute THC and actually developed resistance to aspects of THC-induced social withdrawal (Long et al., 2010a). It is unclear as to why Nrg1cannabinoid interactions are sex-specific. Gender influences the actions of cannabinoids (McGregor and Arnold, 2007) and interactions between gonadal hormones and neuregulin have been demonstrated [(Lacroix-Fralish et al., 2006); but also see Taylor et al., 2011]. Future studies could examine whether Nrg1 expression regulates the modulatory effects of gonadal hormones on cannabinoid receptor sensitivity.

The increased behavioral susceptibility of male $\mathrm{Nrg} 1 \mathrm{HET}$ mice to THC was accompanied by elevated neuronal activation as measured using c-Fos immunohistochemistry (Boucher et al., 2007b). THC selectively increased c-Fos expression in the ventral part of the lateral septum (LSV) of Nrg1 mutants. No corresponding effect was observed in control littermates. Interestingly, drugs, which modulate PPI, whether they are pro-psychotic drugs that impair PPI, or anti-psychotic drugs that facilitate PPI, all increase c-Fos expression in the lateral septum (Sumner et al., 2004). Furthermore, Nrg1 HET mice exhibited a more pronounced enhancement of c-Fos levels in stress-related brain regions (i.e., paraventricular nucleus of hypothalamus and central nucleus of amygdala). In summary, these animal studies provided the very first evidence for an interaction between the schizophrenia risk gene Nrg1 and cannabis and implied that stress and gender may also influence these interactions.
Chronic cannabis use is more relevant in cannabis-induced psychosis than acute exposure. Thus, our team continued this line of research and determined the neuro-behavioral response of Nrg1 mutants to long-term cannabinoid exposure. Nrg1 mutant and control mice were treated chronically with the synthetic CB1 receptor agonist CP 55,940 (Boucher et al., 2011). Nrg1 hypomorphic mice developed tolerance to the hypothermic and locomotor-suppressant effects of CP 55,940 more rapidly than WT mice. Interestingly, tolerance development toward the anxiogenic effects of the cannabinoid was only observed in control mice whereas Nrg1 HETs maintained persistent THC-induced anxiety with repeated CP 55,940 dosing. All mice developed tolerance to the genotype-specific effects of acute CP 55,940 on PPI (i.e., impairment in WT and facilitation in Nrg1 HET mice). Mutant mice showed a selective increase in CP 55,940induced FosB/ $\Delta$ FosB expression in the LSV, which is a marker for long-term neuroadaptive changes. These findings suggest that Nrg1 is not only involved in the acute neuro-behavioral response to cannabinoids but also modulates neuroadaptive responses to long-term cannabinoid challenge. Furthermore, it confirms the LSV as an important brain region for Nrg1-cannabinoid interactions. This could be related to the fact that the lateral septum shares reciprocal projections with the hypothalamus and the amygdala and receives cognitive input from the hippocampus and the prefrontal cortex (Sheehan et al., 2004). These brain areas are important in schizophrenia and are characterized by high expression levels of Nrg1, its main receptor ErbB4 and CB1 (Law et al., 2004; Kofalvi, 2008; Neddens and Buonanno, 2011). Future studies should examine in more detail the role of the LSV in mediating the neuro-behavioral effects of cannabinoids in Nrg1 HET mice and define in particular the involvement of CB1 and ErbB4 receptors.

Human research suggests that adolescence is a time of increased vulnerability to the detrimental effects of cannabis on the development of psychosis (Caspi et al., 2005). Thus, our team exposed adolescent WT and Nrg1 HET mice to chronic THC (Long et al., 2013). Surprisingly, Nrg1 mutants appeared less susceptible to THC-induced suppression of investigative social behaviors than control mice. However, adolescent THC exacerbated the hyperlocomotive phenotype characteristic for adult Nrg1 mutant mice (Karl et al., 2007; Long et al., 2013). Nrg1 deficiency also modulated the effects of adolescent THC on neurotransmitter systems involved in the pathophysiology of schizophrenia. Radioligand binding analyses found genotype-specific THC effects on CB1 expression in the substantia nigra: Nrg1 HET mice exhibited reduced $\mathrm{CB} 1$ levels drug-free whereas $\mathrm{CB} 1$ binding was decreased in WT and increased in Nrg1 mice post THC challenge. Lower CB1 expression levels in the substantia nigra might be responsible for the observed decreased susceptibility of adolescent Nrg1 mutant mice (whereas binding studies in adult Nrg1 HETs found increased levels of CB1 in the same brain region; manuscript currently being submitted). Interestingly, ErbB4 is localized in dopaminergic neurons in the substantia nigra (Abe et al., 2009). Thus, ErbB4 and CB1 might interact in the substantia nigra and thereby regulate the hyper-locomotor phenotype of $\mathrm{Nrgl}$ mutant mice. 
Nrg1 also conferred opposing effects of THC on 5-HT2A receptor expression in the insular cortex and NMDA receptor binding was selectively increased in the hippocampus and cingulate cortex of Nrg1 HET mice (Long et al., 2013) (for a better mechanistic understanding of $\mathrm{Nrg} 1$-THC interaction on NMDA receptor expression in the hippocampus see Spencer et al., 2013).

The cannabis sativa plant is a mix of over 60 different cannabinoids, one being THC, another being CBD, which blocks, or reverses the effects of THC and other psychotropic drugs such as methamphetamine (Long et al., 2010b). Varying levels of THC and CBD in different cannabis strains could modify the consequences of long-term cannabis consumption and also shift the nature of gene-cannabis interactions such as the one reported here for Nrg1. Thus, our team characterized the neuro-behavioral response of Nrg1 HET mice to acute and chronic CBD (Long et al., 2012) to investigate its potentially therapeutic-like effects in animal models for schizophrenia. CBD did not alter schizophrenia-relevant behaviors such as hyperlocomotion or PPI deficits in our Nrg1 HET mouse model (Long et al., 2012). Nevertheless, high dose CBD selectively increased social interaction of $\mathrm{Nrgl}$ mutant mice, which are normally characterized by diminished investigative social behaviors (i.e., social withdrawal) at baseline. Furthermore, chronic CBD also increased GABAA receptor binding in the granular retrosplenial cortex of mutant mice suggesting that $\mathrm{Nrg1}$ may not only modulate neuro-behavioral actions of THC but also of CBD in a task- and brain regionspecific manner. Further research using a variety of CBD doses

\section{REFERENCES}

Abe, Y., Namba, H., Zheng, Y., and Nawa, H. (2009). In situ hybridization reveals developmental regulation of ErbB1-4 mRNA expression in mouse midbrain: implication of ErbB receptors for dopaminergic neurons. Neuroscience 161, 95-110.

Allen, N. C., Bagade, S., McQueen, M. B., Ioannidis, J. P., Kavvoura, F. K., Khoury, M. J., et al. (2008). Systematic meta-analyses and field synopsis of genetic association studies in schizophrenia: the SzGene database. Nat. Genet. 40, 827-834.

Arnold, J. C., Boucher, A. A., and Karl, T. (2012). The yin and yang of cannabis-induced psychosis: the actions of delta 9-tetrahydrocannabinol and cannabidiol in rodent models of schizophrenia. Curr. Pharm. Des. 18, 5113-5130.

Bayer, T. A., Falkai, P., and Maier, W. (1999). Genetic and non-genetic vulnerability factors in schizophrenia: the basis of the "two hit hypothesis". J. Psychiatr. Res. 33, 543-548.

Behan, A. T., Hryniewiecka, M., O'Tuathaigh, C. M., Kinsella, A., Cannon, M., Karayiorgou, M., et al. (2012). Chronic adolescent exposure to delta-9tetrahydrocannabinol in COMT mutant mice: impact on indices of dopaminergic, endocannabinoid and GABAergic pathways. Neuropsychopharmacology 37, 1773-1783.

Boucher, A. A., Arnold, J. C., Duffy, L., Schofield, P. R., Micheau, J., and Karl, T. (2007a). Heterozygous neuregulin 1 mice are more sensitive to the behavioural effects of Delta9-tetrahydrocannabinol. Psychopharmacology (Berl.) 192, 325-336. T., Micheau, J., McGregor, I. S., and Arnold, J. C. (2007b). Heterozygous neuregulin 1 mice display greater baseline and Delta (9) - tetrahydrocannabinol-induced c-Fos expression. Neuroscience 149, 861-870.

Boucher, A. A., Hunt, G. E., Micheau, J., Huang, X., McGregor, I. S., Karl, T., et al. (2011). The schizophrenia susceptibility gene neuregulin 1 modulates tolerance to the effects of cannabinoids. Int. J. Neuropsychopharmacol. 14, 631-643.

Caspi, A., and Moffitt, T. E. (2006). Gene-environment interactions
Boucher, A. A., Hunt, G. E., Karl,

thereby considering dose and age-effects will address the issue of varying or even opposing effects of different cannabinoids more comprehensively.

In summary, the transmembrane domain Nrg1 mouse model has enabled the detailed analysis of acute vs. chronic effects of cannabinoids at different stages of brain development. $\mathrm{Nrgl}$ modulated the behavioral sensitivity of mice to cannabinoids differentially during adolescence and adulthood providing evidence for a role of Nrg1-cannabis interactions in schizophrenia. Furthermore, insights into the molecular and neurobiological mechanisms of Nrg1-cannabinoid interactions (involvement of CB1, 5-HT2A and NMDA receptors in particular) would not have been possible without utilizing these mouse mutants. Future research will extend on our initial findings and address sex specificity and the opposite effects of CB1 stimulation in adolescence (Nrg1 mutant less susceptible) and adulthood (Nrg1 mutants more susceptible) in greater detail. Finally, models of cannabinoid addiction should be considered given the significant comorbidity of schizophrenia and drug dependence.

\section{ACKNOWLEDGMENTS}

Tim Karl is supported by the Schizophrenia Research Institute utilizing infrastructure funding from NSW Ministry of Health, a project grant (1003886) and a career development fellowship (1045643) from the National Health and Medical Research Council, and a research grant from the Motor Neuron Disease Research Institute of Australia (Mick Rodger Benalla MND Research Grant).

in psychiatry: joining forces with neuroscience. Nat. Rev. Neurosci. 7, 583-590.

Caspi, A., Moffitt, T. E., Cannon, M. McClay, J., Murray, R., Harrington, H., et al. (2005). Moderation of the effect of adolescent-onset cannabis use on adult psychosis by a functional polymorphism in the catechol-O-methyltransferase gene: longitudinal evidence of a gene $\mathrm{X}$ environment interaction. Biol. Psychiatry 57, 1117-1127.

Chesworth, R., Downey, L., Logge, W., Killcross, S., and Karl, T. (2012a). Cognition in female transmembrane domain neuregulin 1 mutant mice. Behav. Brain Res. 226, 218-223.

Chesworth, R., Yulyaningsih, E., Cappas, E., Arnold, J., Sainsbury, A., and Karl, T. (2012b). The response of neuregulin 1 mutant mice to acute restraint stress. Neurosci. Lett. 515, 82-86.

Desbonnet, L., Waddington, J. L., and O’Tuathaigh, C. M. (2009). Mutant models for genes associated with schizophrenia. Biochem. Soc. Trans. 37, 308-312.

Duffy, L., Cappas, E., Lai, D., Boucher, A. A., and Karl, T. (2010). Cognition in transmembrane domain neuregulin 1 mutant mice. Neuroscience 170, 800-807.

Duffy, L., Cappas, E., Scimone, A., Schofield, P. R., and Karl, T. (2008). Behavioral profile of a heterozygous mutant mouse model for EGFlike domain neuregulin 1. Behav. Neurosci. 122, 748-759.

Geyer, M. A., Krebs-Thomson, K., Braff, D. L., and Swerdlow, N. R. (2001). Pharmacological studies of prepulse inhibition models of sensorimotor gating deficits in schizophrenia: a decade in review. Psychopharmacology (Berl.) 156, 117-154.

Han, S., Yang, B. Z., Kranzler, H. R., Oslin, D., Anton, R., Farrer, L. A. et al. (2012). Linkage analysis followed by association show NRG1 associated with cannabis dependence in African Americans. Biol. Psychiatry 72, 637-644.

Harrison, P. J., and Law, A. J. (2006). Neuregulin 1 and schizophrenia: genetics, gene expression, and neurobiology. Biol. Psychiatry 60, 132-140.

Henquet, C., Murray, R., Linszen, D., and Van Os, J. (2005). The environment and schizophrenia: the role of cannabis use. Schizophr. Bull. 31, 608-612. 
Henquet, C., Rosa, A., Krabbendam, L., Papiol, S., Fananas, L., Drukker, M., et al. (2006). An experimental study of catechol-o-methyltransferase Val158Met moderation of delta9-tetrahydrocannabinol-induced effects on psychosis and cognition. Neuropsychopharmacology 31, 2748-2757.

Karl, T., Burne, T. H., Van Den Buuse, M., and Chesworth, R. (2011). Do transmembrane domain neuregulin 1 mutant mice exhibit a reliable sensorimotor gating deficit? Behav. Brain Res. 223, 336-341.

Karl, T., Duffy, L., Scimone, A., Harvey, R. P., and Schofield, P. R. (2007). Altered motor activity, exploration and anxiety in heterozygous neuregulin 1 mutant mice: implications for understanding schizophrenia. Genes. Brain Behav. 6, 677-687.

Kofalvi, A. (2008). Cannbinoids and the Brain. New York, NY: Springer.

Lacroix-Fralish, M. L., Tawfik, V. L., Nutile-McMenemy, N., Harris, B. T., and Deleo, J. A. (2006). Differential regulation of neuregulin 1 expression by progesterone in astrocytes and neurons. Neuron Glia Biol. 2, 2227-2234.

Law, A. J., Shannon Weickert, C., Hyde, T. M., Kleinman, J. E., and Harrison, P. J. (2004). Neuregulin1 (NRG-1) mRNA and protein in the adult human brain. Neuroscience 127, 125-136.

Long, L. E., Chesworth, R., Arnold, J. C., and Karl, T. (2010a). A follow-up study: acute behavioural effects of Delta(9)-THC in female heterozygous neuregulin 1 transmembrane domain mutant mice. Psychopharmacology (Berl.) 211, 277-289.

Long, L. E., Chesworth, R., Huang, X. F., McGregor, I. S., Arnold, J. C., and Karl, T. (2010b). A behavioural comparison of acute and chronic Delta9-tetrahydrocannabinol and cannabidiol in C57BL/6JArc mice.
Int. J. Neuropsychopharmacol. 13, 861-876.

Long, L. E., Chesworth, R., Huang, X. F., McGregor, I. S., Arnold, J. C., and Karl, T. (2013). Transmembrane domain Nrgl mutant mice show altered susceptibility to the neurobehavioural actions of repeated THC exposure in adolescence. Int. J. Neuropsychopharmacol. 16, 163-175.

Long, L. E., Chesworth, R., Huang, X. F., Wong, A., Spiro, A., McGregor, I. S., et al. (2012). Distinct neurobehavioural effects of cannabidiol in transmembrane domain neuregulin 1 mutant mice. PLoS ONE 7:e34129. doi: 10.1371/ journal.pone.0034129

McGregor, I. S., and Arnold, J. C. (2007). Cannabis reward: biased towards the fairer sex? $\mathrm{Br}$. J. Pharmacol. 152, 562-564.

Mei, L., and Xiong, W. C. (2008). Neuregulin 1 in neural development, synaptic plasticity and schizophrenia. Nat. Rev. Neurosci. 9, 437-452.

Munafo, M. R., Thiselton, D. L. Clark, T. G., and Flint, J. (2006). Association of the NRG1 gene and schizophrenia: a meta-analysis. Mol. Psychiatry 11, 539-546.

Neddens, J., and Buonanno, A. (2011). Expression of the neuregulin receptor ErbB4 in the brain of the rhesus monkey (Macaca mulatta). PLOS ONE 6:e27337. doi: 10.1371/ journal.pone.0027337

O’Tuathaigh, C. M., Hryniewiecka, M., Behan, A., Tighe, O., Coughlan, C., Desbonnet, L., et al. (2010). Chronic adolescent exposure to Delta-9tetrahydrocannabinol in COMT mutant mice: impact on psychosisrelated and other phenotypes. Neuropsychopharmacology 35, 2262-2273.

Powell, C. M., and Miyakawa, T. (2006). Schizophrenia-relevant behavioural testing in rodent models: a uniquely human disorder? Biol. Psychiatry 59, 1198-1207.

Rapoport, J. L., Addington, A. M. Frangou, S., and Psych, M. R. (2005). The neurodevelopmental model of schizophrenia: update 2005. Mol. Psychiatry 10, 434-449.

Sanders, A. R., Duan, J., Levinson, D. F., Shi, J., He, D., Hou, C., et al. (2008). No significant association of 14 candidate genes with schizophrenia in a large European ancestry sample: implications for psychiatric genetics. Am. J. Psychiatry 165, 497-506.

Sheehan, T. P., Chambers, R. A., and Russell, D. S. (2004). Regulation of affect by the lateral septum: implications for neuropsychiatry. Brain Res. Brain Res. Rev. 46, 71-117.

Spencer, J. R., Darbyshire, K. M. Boucher, A. A., Kashem, M. A., Long, L. E., McGregor, I. S., et al. (2013). Novel molecular changes induced by Nrgl hypomorphism and Nrg1-cannabinoid interaction in adolescence: a hippocampal proteomic study in mice. Front. Cell. Neurosci. 7:15. doi: 10.3389/fncel.2013.00015

Stadelmann, A. M., Roser, P., Arning, L., Gallinat, J., Epplen, J. T., and Juckel, G. (2010). Acute effects of delta9-tetrahydrocannabinol on the auditory evoked mismatch negativity are modulated by the NRG1 gene. Pharmacopsychiatry 43, 194-195.

Stefansson, H., Sigurdsson, E., Steinthorsdottir, V., Bjornsdottir, S., Sigmundsson, T., Ghosh, S., et al. (2002). Neuregulin 1 and susceptibility to schizophrenia. Am. J. Hum. Genet. 71, 877-892.

Sumner, B. E., Cruise, L. A., Slattery, D. A., Hill, D. R., Shahid, M., and Henry, B. (2004). Testing the validity of $\mathrm{c}$-fos expression profiling to aid the therapeutic classification of psychoactive drugs. Psychopharmacology (Berl.) 171, 306-321.
Taylor, S. B., Markham, J. A., Taylor, A. R., Kanaskie, B. Z., and Koenig, J. I. (2011). Sex-specific neuroendocrine and behavioral phenotypes in hypomorphic Type II Neuregulin 1 rats. Behav. Brain Res. 224, 223-232.

Tosato, S., Dazzan, P., and Collier, D. (2005). Association between the neuregulin 1 gene and schizophrenia: a systematic review. Schizophr. Bull. 31, 613-617.

Van Den Buuse, M., Wischhof, L., Lee, R. X., Martin, S., and Karl, T. (2009) Neuregulin 1 hypomorphic mutant mice: enhanced baseline locomotor activity but normal psychotropic drug-induced hyperlocomotion and prepulse inhibition regulation. Int. J. Neuropsychopharmacol. 12, 1383-1393.

Van Os, J., Kenis, G., and Rutten, B. P. (2010). The environment and schizophrenia. Nature 468, 203-212.

Conflict of Interest Statement: The authors declare that the research was conducted in the absence of any commercial or financial relationships that could be construed as a potential conflict of interest.

Received: 16 November 2012; accepted: 10 February 2013; published online: 26 February 2013.

Citation: Karl T and Arnold JC (2013) What does a mouse tell us about neuregulin 1-cannabis interactions? Front. Cell. Neurosci. 7:18. doi: 10.3389/fncel. 2013.00018

Copyright (c) 2013 Karl and Arnold. This is an open-access article distributed under the terms of the Creative Commons Attribution License, which permits use, distribution and reproduction in other forums, provided the original authors and source are credited and subject to any copyright notices concerning any third-party graphics etc. 